\title{
Boundary layer separation by a step in surface temperature
}

\author{
F. MÉNDEZ and C. TREVIÑO
}

and

A. LIÑÁN

\begin{abstract}
The free-interaction influence of thermal expansion process in the boundary layer gas flow is analysed in this paper, using the formalism of the Triple-Deck theory. The physical model considered herein is the forced convection of a gas flowing over a flat plate subject to a step change in the surface temperature, taking place at a certain distance from the leading edge. There is a fundamental parameter $T_{\mathrm{w}}$, defined as the ratio of the wall temperature to the free stream temperature. For values of $T_{\mathrm{w}}$ close to one, the governing equations can be linearized and solved with the aid of the Fourier transform method. However, for values of this parameter not close to one, a numerical treatment is required to solve the governing equations. Using finite-difference methods, the numerical results for the pressure, skin friction, thickness displacement and Nusselt number are presented for different values of $T_{\mathrm{w}}$. Finally, for a critical value of this temperature ratio, the boundary layer separates.
\end{abstract}

\section{INTRODUCTION}

SINCE the classic works by Stewartson and Williams [1], Messiter [2] and Stewartson [3] for the study of the influence on the drag coefficient of a finite length flat plate, using a three layer structure, there has been a renewed interest in applying it to a host of other basic flow situations, involving normally small disturbances of different nature. In particular, the presence of some disturbances on the plate surface, generates an interaction with the outer inviscid flow, producing longitudinal pressure gradients having an important influence on the fluid close to the wall where the convective terms are small. It has been demonstrated that the Triple-Deck structure can be utilized to explain correctly those flows and to predict boundary layer separation. A comprehensive review on this subject is given by Smith [4], where the same structural argument of the Triple-Deck theory can be applied immediately to a wide variety of other incompressible and compressible problems. Particular studies of the incompressible case come from Stewartson [5] for a corner, the extensively studied case for a hump or dent mainly analysed by Smith and Merkin [6], Veldman and Dijkstra [7], the slot-injection by Napolitano and Messick [8]. The compressible Triple-Deck structure has also received considerable attention in the literature for subsonic and supersonic flows $[1,9]$. However, the majority of these works consider the disturbance associated with some kind of dynamical or geometrical discontinuity like the velocity value as boundary condition or the finite hump on a flat plate and similar effects. On the other hand, Messiter and Liñan [10] study the problem of the vertical plate in a laminar free convection flow. In particular, their analyses consider the flow near a discontinuity in plate temperature and show that a free convection interaction of the same general type occurs near a jump discontinuity in the prescribed plate temperature. This analysis can be simplified considerably because the boundary conditions for the velocity are continuous and an analytical solution for the local pressure distribution can be obtained. Recently, Zeytounian [11] has proposed to study the incompressible flow in a flat plate subject to a step temperature, in a finite interval of the length of the plate.

In the present work, the focus is on investigating the fluid-mechanical structure of a gaseous boundary layer flow produced by the thermal expansion process using the Triple-Deck theory. This process is generated by abruptly increasing at a given distance the plate temperature from that of the free stream to a higher value. This problem has been studied analytically and numerically by solving the multi-layer governing equations, obtaining a boundary layer separation for a critical value of the temperature ratio.

\section{GOVERNING EQUATIONS}

The physical model analysed is the following. We consider a flat plate aligned parallel to a uniform free-stream gas flow which has a uniform free-stream velocity $u_{\infty}^{*}$ and temperature $T_{\infty}^{*}$. We will assume that 


\section{NOMENCLATURE}

$A(\bar{x})$ nondimensional displacement thickness

$A_{\mathrm{i}}(\bar{x})$ Airy function

$\hat{A}_{0}(\bar{x})$ nondimensional linear displacement thickness

$B(w)$ complex constant

$\mathbb{B}$ constant of relaxation

$C_{i j} \quad$ matrix related with the interaction law

$c_{\mathrm{p}} \quad$ specific heat of the gas

$F_{0} \quad$ nondimensional stream function

$G_{0} \quad$ nondimensional temperature distribution

$H(\vec{x})$ Heaviside step function

$i \quad$ imaginary number

Im $\omega$ imaginary part of $\omega$

$k$ thermal conductivity

$\mathbb{K}$ constant depending of Prandtl number, $9^{1 / 3} \Gamma(2 / 3) / \Gamma(1 / 3) \operatorname{Pr}^{1 / 3}$

$l^{*} \quad$ longitudinal distance between the leading edge and the temperature step

$N u_{\mathrm{L}} \quad$ Nusselt number based on the Lighthill approximation

$\mathrm{Nu} \quad$ Nusselt number, defined by $N u=$ $-\theta_{\mathrm{w}}\left(1+\lambda^{-5 / 4} R^{-3 / 8} \bar{x}\right) /\left(T_{\mathrm{w}}-1\right) \lambda^{-3 / 4} R^{-5 / 8}$ $\times \partial T /\left.\partial \bar{y}\right|_{\bar{y}=0}$

$\operatorname{Pr} \quad$ Prandtl number, $\mu_{\propto}^{*} c_{\mathrm{p}} / k$

$p_{\infty}^{*} \quad$ pressure far from the plate

$p^{*} \quad$ pressure distribution

$p$ nondimensional pressure, $p^{*}-p_{\infty}^{*} / \rho_{\infty}^{*} u_{\infty}^{2}$

$\hat{p}_{0} \quad$ nondimensional linear pressure

$\tilde{p} \quad$ nondimensional pressure at the lower-deck

$R \quad$ Reynolds number, $u_{\infty}^{*} l^{*} / \nu_{\infty}^{*}$

$R^{*} \quad$ ideal gas constant

$R_{\text {j }} \quad$ vector related with the interaction law

$T_{\infty}^{*} \quad$ temperature far from the plate

$T_{\mathrm{w}}^{*} \quad$ wall temperature

$T^{*} \quad$ temperature distribution

$T_{\mathrm{w}} \quad$ nondimensional temperature parameter, $T_{\mathrm{w}}^{*} / T_{\infty}^{*}$

$T$ nondimensional temperature, $T^{*} / T_{\infty}^{*}$

$\hat{T}_{0} \quad$ nondimensional linear temperature up to terms of order $\theta_{\mathrm{w}}$

$\hat{T}_{\mathrm{I}} \quad$ nondimensional linear temperature up to terms of order $\theta_{\mathrm{w}}^{2}$

$\pi$ change of variable to follow the Box-Keller method, equation (66)

$u_{\infty}^{*} \quad$ velocity at the $x$ direction far from the plate

$u^{*} \quad$ velocity distribution at the $x$ direction

$u \quad$ nondimensional velocity at the $x$ direction, $u^{*} / u_{\infty}^{*}$

$\bar{u} \quad$ nondimensional lower-deck velocity at the $x$ direction

$\hat{u}_{0} \quad$ nondimensional linear longitudinal velocity

U change of variable to follow the Box-Keller method, equation (63) $v^{*} \quad$ velocity at the $y$ direction

$v$ nondimensional velocity at the $y$

direction, $v^{*} / u_{\infty}^{*}$

$\bar{v} \quad$ nondimensional lower-deck velocity at the $y$ direction

$\hat{v}_{0} \quad$ nondimensional linear transversal velocity

$\checkmark \quad$ change of variable to follow the Box-Keller method, equation (64)

$x^{*} \quad$ longitudinal coordinate measured from the leading edge, Fig.

$x$ nondimensional longitudinal coordinate, $x^{*} / l^{*}$

$\bar{x} \quad$ longitudinal scale within the lowerdeck, equation (7)

$\bar{x}_{0} \quad$ initial value for $\bar{x}$ to complete the numerical calculations

$y^{*} \quad$ transversal coordinate measured from the leading edge, Fig. 1

$y$ nondimensional transversal coordinate, $y^{*} / l^{*}$

$\bar{y} \quad$ transversal scale within the lowerdeck, equation (7)

$\bar{y}_{0} \quad$ initial value for $\bar{y}$ to complete the numerical calculations

$\bar{y}_{\mathrm{e}} \quad$ edge value of the $\bar{y}$ scale

$Y$ transversal coordinate of the boundary layer, $y=R^{-4 / 8} Y$

$z \quad$ modified longitudinal coordinate, $z=\vec{x}^{1 / 3}$.

Greek symbols

$\delta \quad$ Dirac delta function

$\eta \quad$ non-similar variable, equation (15)

$\theta \quad$ nondimensional temperature

$\theta_{\mathrm{w}} \quad$ temperature parameter defined by $T_{\mathrm{w}}-1$

$\mu_{\infty}^{*} \quad$ viscosity at the free stream of the gas

$\mu^{*} \quad$ viscosity coefficient

$\mu$ nondimensional viscosity coefficient, $\mu^{*} / \mu_{\infty}^{*}$

$v_{\infty}^{*} \quad$ kinematic viscosity at the free stream of the gas, $\mu_{\infty}^{*} / \rho_{\infty}^{*}$

$\Xi(P r)$ constant depending on Prandtl number, equation (21)

$\rho_{\infty}^{*} \quad$ density function at the free stream of the gas

$\rho^{*} \quad$ density of the gas

$\rho_{\mathrm{w}} \quad$ wall density

$\sigma \quad$ stretching variable on the linear analysis, $\bar{y}=\sigma / \operatorname{Pr}^{1 / 3}$

$\tau$ nondimensional skin friction

$\hat{\tau}_{0} \quad$ nondimensional linear skin friction

$\phi \quad$ high order function to determine $\hat{T}_{1}$

$\psi_{0} \quad$ nonsimilar stream function defined by equation (15) 

equation (63) Fourier frequency.

\section{Subscripts}

B Blasius profile

e outer edge of the lower-deck

F Fourier variable

w value of the variables at the wall value of the variables at the free stream.

\section{Superscripts}

bar to denote linear variables

bar to denote the lower-deck compressible variables

- bar to denote the lower-deck incompressible variables

* asterisk to denote the dimensional physical variables. the free-stream gas temperature is equal to the wall temperature. However, at a certain distance from the leading edge, the flat plate temperature is suddenly changed to a uniform value $T_{w}^{*}$ greater than the gas temperature. Thus, the heat transfer will always occur from the flat plate to the gas, causing logically an expansion process in the gas flow. The physical situation is shown in Fig. 1. Specifically, the main features of the flow can be modified substantially if we analyse regions very close to the step temperature where the strong gas expansion causes the retention of the pressure gradients in the governing equations due to the free interaction phenomena. The displacement thickness evolution, for this region, changes in an important way due to the very strong expansion process. It is well known, that the TripleDeck structure can be described by three layers (upper, main and lower deck) each of them satisfying appropriated governing equations [4]. In this case the lower-deck is the only nonisothermal layer, which is produced by the altered boundary conditions at the flat plate, due to the drastic change in the wall temperature. Because the equations of motion for the upper and main decks permit simple analytical solutions, we must concentrate herein on the problem of the lower-deck. The starting point in order to arrive at the lower-deck equations comes from considering the compressible Navier-Stokes equations together with an appropriate energy equation for two-dimensional forced flow. The nondimensional governing equations, assuming completely negligible Eckert number (low Mach number flows), are given by

$$
\begin{gathered}
\frac{\partial(\rho u)}{\partial x}+\frac{\partial(\rho v)}{\partial y}=0 \\
\rho u \frac{\partial u}{\partial x}+\rho v \frac{\partial u}{\partial y}=-\frac{\partial p}{\partial x}+\frac{1}{R}\left\{\frac { \partial } { \partial x } \mu \left[2 \frac{\partial u}{\partial x}\right.\right. \\
\left.\left.-\frac{2}{3}\left\{\frac{\partial u}{\partial x}+\frac{\partial v}{\partial y}\right\}\right]+\frac{\partial}{\partial y} \mu\left[\frac{\partial u}{\partial y}+\frac{\partial v}{\partial x}\right]\right\} \\
\rho u \frac{\partial v}{\partial x}+\rho v \frac{\partial v}{\partial y}=-\frac{\partial p}{\partial y}+\frac{1}{R}\left\{\frac { \partial } { \partial y } \mu \left[2 \frac{\partial u}{\partial y}\right.\right. \\
\left.\left.-\frac{2}{3}\left\{\frac{\partial u}{\partial x}+\frac{\partial v}{\partial y}\right\}\right]+\frac{\partial}{\partial x} \mu\left[\frac{\partial u}{\partial y}+\frac{\partial v}{\partial x}\right]\right\}
\end{gathered}
$$

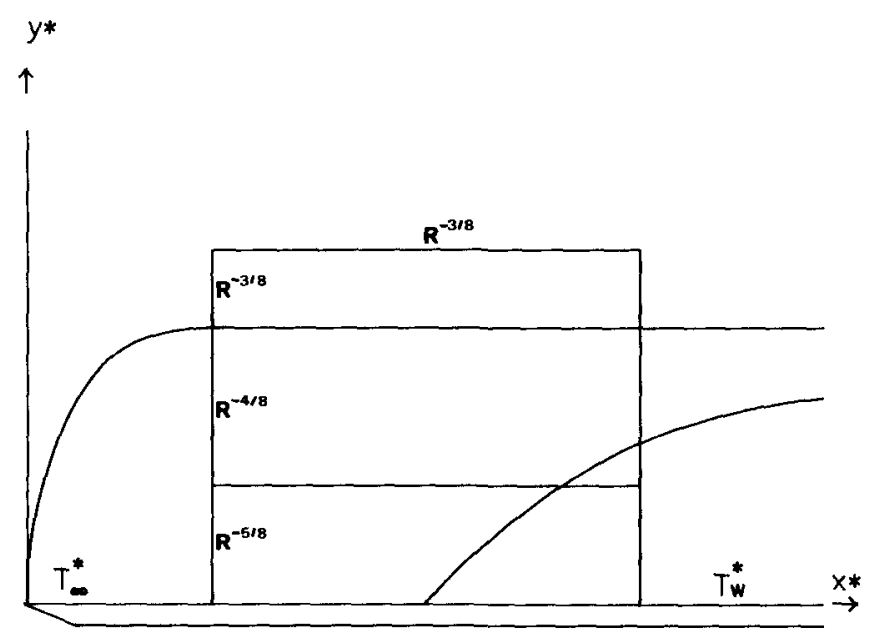

FIG. 1. Sketch of the thermal expansion interaction process. 
$\rho u \frac{\partial T}{\partial x}+\rho v \frac{\partial T}{\partial y}=\frac{1}{R \operatorname{Pr}}\left[\frac{\partial}{\partial x}\left(\mu \frac{\partial T}{\partial x}\right)+\frac{\partial}{\partial y}\left(\mu \frac{\partial T}{\partial y}\right)\right]$.

The nondimensional variables and the corresponding symbols used in this work are given in the Nomenclature. In addition to the above equations, we need an equation of state. In this case for simplicity we take an ideal gas law given by $p=\rho R^{*} T$. Similarly, if we assume for simplicity that the gas viscosity increases linearly with temperature (ChapmanRubesin parameter, $C=1$ ), both relations are given in nondimensional form as :

$$
\rho T=1, \quad \rho \mu=1 .
$$

In the above equation (5) the variation in pressure in the state equation has been neglected, which is justified for these low Mach number flows. The origin of the two-dimensional Cartesian coordinate system is taken to be at the leading edge of the flat plate where $x$ and $y$ represent the longitudinal and transversal coordinates to the plate, respectively. Also, in the above equations, $P r$ and $R$ represent the Prandtl and Reynolds numbers defined by $\operatorname{Pr}=\mu_{\infty}^{*} C_{\mathrm{p}} / k$ and $R=u_{\infty}^{*} l^{*} / v_{\infty}^{*}$, respectively. We also assume that the Prandtl number is constant. The boundary conditions required to complete the problem statement are specified by the following relationships:

$$
\begin{gathered}
\text { at } y=0: \quad u=v=0, \quad T=1 \quad \text { for } x \leqslant 1 \text { (6a) } \\
T=T_{\mathrm{w}} \text { for } x>1 \\
\text { at } x=0 ; \quad y>0: \quad u=T=1 .
\end{gathered}
$$

Finally, far away from the flat plate, we have

$$
x^{2}+y^{2} \rightarrow \infty: \quad u \rightarrow 1, T \rightarrow 1
$$

Clearly, for fixed values of $R$ and $P r$, the analytical model depends only on a parameter $T_{\mathrm{w}}$ which is given by the ratio of the wall temperature to the free stream temperature, $T_{\mathrm{w}}=T_{\mathrm{w}}^{*} / T_{\infty}^{*}$ and the specified values of it dictates the procedure to solve the governing equations.

\section{Lower-deck equations}

In order to consider those regions where the thermal expansion becomes considerable it is required to introduce the adequate Triple-Deck structure scaling. It can be shown [4] that in regions close to the point where the step change in temperature takes place, the value of the pressure in higher order equations can take an irregular form. So, when this point is approached, the induced pressure gradient eventually becomes comparable with the leading order inertial terms when $x-1=0\left(R^{-3 / 8}\right)$. Therefore, in order to analyse the effects in the lower-deck caused by a thermal expansion at the field flow, the following new expansions and scaling are introduced:

$$
\begin{gathered}
u=\lambda^{1 / 4} R^{-1 / 8} \tilde{u}+\cdots \\
v=\lambda^{3 / 4} R^{-3 / 8} \tilde{v}+\cdots \\
p=\lambda^{1 / 2} R^{-1 / 4} \tilde{p}+\cdots \\
x-1=\lambda^{-5 / 4} R^{-3 / 8} \tilde{x}, \quad y=\lambda^{-3 / 4} R^{-5 / 8} \tilde{y}
\end{gathered}
$$

where $\lambda=0.332$. In this form, the above governing equations can be reduced to

$$
\begin{gathered}
\frac{\partial}{\partial \tilde{x}} \rho \tilde{u}+\frac{\partial}{\partial \tilde{y}} \rho \tilde{v}=0 \\
\rho \tilde{u} \frac{\partial \tilde{u}}{\partial \tilde{x}}+\rho \tilde{v} \frac{\partial \tilde{u}}{\partial \tilde{y}}=-\frac{\partial \tilde{p}}{\partial \tilde{x}}+\frac{\partial}{\partial \tilde{y}}\left(\mu \frac{\partial \tilde{u}}{\partial \tilde{y}}\right) \\
\frac{\partial \tilde{p}}{\partial \tilde{y}}=0\left(R^{-4 / 8}\right) \\
\rho \tilde{u} \frac{\partial T}{\partial \tilde{x}}+\rho \tilde{v} \frac{\partial T}{\partial \tilde{y}}=\frac{1}{\operatorname{Pr} \partial \tilde{y}}\left(\mu \frac{\partial T}{\partial \tilde{y}}\right) .
\end{gathered}
$$

The above compressible governing equations should be completed with appropriate boundary conditions for the lower-deck given by

at $\tilde{y}=0: \quad \tilde{u}=\tilde{v}=0, \quad T=1+\left(T_{\mathrm{w}}-1\right) H(\tilde{x})$

where $H(\tilde{x})$ represents the Heaviside step function.

As $\tilde{x} \rightarrow-\infty$, the solution must match with the Blasius solution given by $\tilde{u} \rightarrow u_{\mathrm{B}}(Y)$, when $Y$ is small. Here, $Y$ is the inner-variable normally used to analyse the classical boundary layer and it is related with $y$ through this relationship $y=R^{-4 / 8} Y$ or also, in terms of the transversal lower-deck variable as $Y=R^{-1 / 8} \tilde{y}$. Similarly, when $\tilde{x} \rightarrow-\infty$ the temperature must tend to that of the free stream, i.e. $T \rightarrow 1$ as $\tilde{x} \rightarrow-\infty$. In the region $\tilde{x} \rightarrow 0$ of order $\left(\tilde{x} \sim R e^{-3 / 4}\right)$ there is another thin region (not resolved by the Triple-Deck structure) called by Stewartson 'Central Region' [3], where the full Navier-Stokes equations are applicable. The influence of the structure of this zone is not primordial and its presence is dictated by jump-conditions in the Triple-Deck coordinates at $x=0$. Then, without considering longitudinal heat transfer mechanism $\left(|\tilde{x}| \gg R e^{-3 / 4}\right) ; T=1$ for $\tilde{x}<0$. On the other hand, for large values of $\tilde{y}$, the expansions (7) must be matched with the main deck. However, we shall not discuss here the details, to be found elsewhere [3]. The corresponding boundary conditions are given by:

$$
u \sim \tilde{y}+A(\tilde{x}), \text { for } \tilde{y} \rightarrow \infty
$$

where $A(\tilde{x})$ represents the displacement thickness with sign changed and also the velocity slip at the base of the main-deck, corresponding to the inviscid perturbation of the upstream Blasius solution by the induced pressure gradient. In addition, a boundary condition of the temperature is required to complete this matching with the main-deck and is given by:

$$
T \sim 1, \text { for } \tilde{y} \rightarrow \infty \text {. }
$$

This is because in the main-deck $\rho u_{\mathrm{B}} \partial T / \partial \tilde{x}=$ $0\left(R^{-1 / 8}\right)$, thus $T(\tilde{x}, Y)=f(Y)$. But similarly, 
when the function $T(\tilde{x}, \tilde{y})$ is matched with the upper-deck the only possibility is that $T=1$, which is dictated by the imposed boundary condition at the free-stream. Due to the elliptic nature of the problem, an additional boundary condition is required for values of $\tilde{x} \rightarrow \infty$ which matches with the modified Blasius solution for a compressible boundary layer heated by a step change in the temperature of the flat plate. From the point of view of the classical boundary layer theory, the neighborhood of the step change on the flat plate, i.e. when $x \rightarrow 1$ has been analysed by different authors as Sparrow and Yu [12], Cebeci and Bradshaw [13], Lighthill [14] and the analytical treatment follows that of Goldstein's analysis [15] of the near-wake of the aligned flat plate. In these works it is shown that continuation of the Blasius solution into the wake is possible by introducing an irregularity just downstream of the trailing edge. Furthermore, provided separation does not occur at $x-1<0$, the only singularity in the solution occurs at $x=1$ and it is due to the change in boundary conditions [3]. The transition of the solution from $x=1^{*}$, to $x=1^{+}$is achieved by the Rott and Hakkinen [16] modification of the Goldstein wake and in our case is given by

$$
\begin{gathered}
\eta=\frac{Y}{(x-1)^{1 / 3}}, \quad \psi_{0}=(x-1)^{2 / 3} F_{0}+\cdots \\
\frac{\partial p}{\partial x} \sim b(x-1)^{-1 / 3}, \quad T(x, Y)=G_{0}(\eta)+\cdots
\end{gathered}
$$

for $x \rightarrow 1$ and $x-1 \gg R^{-3 / 8}$. The velocity and temperature fields in the neighborhood of the step region satisfy to the lowest order, the following equations:

$$
\begin{gathered}
F_{0}^{\prime \prime}+{ }_{3}^{2} F_{0} F_{0}^{\prime \prime}-{ }_{3}^{1} F_{0}^{\prime 2}={ }_{3}^{2} b \\
\frac{1}{P r} G_{0}^{\prime \prime}+\frac{2}{3} F_{0} G_{0}^{\prime}=0
\end{gathered}
$$

where primes denote derivatives with respect to $\eta$. The boundary conditions are then given by

$$
\begin{aligned}
& \text { at } \eta=0: \quad F_{0}=F_{0}^{\prime}=0, \quad G_{0}=T_{\mathrm{w}} \\
& \text { at } \eta \rightarrow \infty: \quad F_{0} \rightarrow \frac{0.332}{2} \eta^{2}+\eta \int_{0}^{\infty}\left(G_{0}-1\right) \mathrm{d} \eta+\cdots, \\
& \quad G_{0} \rightarrow 1 . \quad(18)
\end{aligned}
$$

In the above equations $b$ should be a positive quantity which is evaluated numerically. Because it might be expected that the pressure gradient in $x-1<0$ is disfavorable, $\partial p / \partial x$ represents the so-called 'vorticityinduced' pressure gradient [16].

It should be noted that if we use the approximation $F_{0} \sim 0.332 \eta^{2} / 2$ for the energy equation (17), it can be integrated and after some manipulations we obtain :

$$
G_{0}=T_{\mathrm{w}}+0.7764\left(1-T_{\mathrm{w}}\right) \int_{0}^{\left(0332 P_{r / 9)^{1 / 3}}\right.} \mathrm{e}^{-u^{3}} \mathrm{~d} u .
$$

These solutions for $F_{0}$ and $G_{0}$ are required to initialize the numerical problem of the lower-deck. In this sense and after some manipulations, it can be demonstrated that the displacement thickness and the pressure are given by the following expressions:

$$
\begin{aligned}
& A(x)=-\Xi(\operatorname{Pr})\left(T_{\mathrm{w}}-1\right)(x-1)^{1 / 3} \\
& p(x)=-\Xi(\operatorname{Pr})\left(T_{\mathrm{w}}-1\right)(x-1)^{-2 / 3}
\end{aligned}
$$

for $x \rightarrow 1^{+}$and $\Xi(\operatorname{Pr})$ is a Prandtl's function given by :

$$
\Xi(P r)=\frac{\int_{0}^{\infty} \int_{(P r / 9)^{1 / 3}}^{\infty} \exp \left(-v^{3}\right) \mathrm{d} v \mathrm{~d} u}{\int_{0}^{\infty} \exp \left(-v^{3}\right) \mathrm{d} v} .
$$

Complementary, it can be shown that the corresponding expressions for the displacement thickness and pressure for $x \rightarrow 1^{-}$are given as

$$
\begin{aligned}
& A(x)=0\left((x-1)^{1 / 3}\right) \\
& p(x)=\frac{2\left(T_{w}-1\right)}{3 \sqrt{3}} \frac{\Xi(P r)}{[-(x-1)]^{2 / 3}} .
\end{aligned}
$$

The relationships given by equations (20) and (22) are needed to complete the boundary conditions upstream and downstream for the thermal expansion process.

Finally, an interaction condition, which is derived by the thin airfoil linearized theory is required in order to match with the upper-deck solution and corresponds to the well-known pressure-displacement relation [3]:

$$
\tilde{p}(\tilde{x})=\frac{1}{\pi} \oint_{-\infty}^{\infty} \frac{A^{\prime}\left(\tilde{x}_{1}\right) \mathrm{d} \tilde{x}_{1}}{\tilde{x}-\tilde{x}_{1}}
$$

where in the last equation prime means derivative with respect to the dummy variable $\tilde{x}_{1}$ and the integral represents the principal value of the Cauchy-Hilbert integral. The set of equations define the thermal expansion problem within the longitudinal scale $x-1=\hat{\lambda}^{-5 / 4} R^{-3 / 8} \tilde{x}$. However these equations can be simplified even more by employing the HowarthDorodnitzyn transformation [17] giving an equivalent set of incompressible governing equations. Therefore, defining the following nondimensional variables :

$$
\bar{x}=\tilde{x}, \quad \bar{y}=\int^{\bar{y}} \rho(\tilde{x}, \tilde{y}) \mathrm{d} \tilde{y} \quad \rho \bar{u}=\rho \tilde{u} \quad \bar{v}=\rho \tilde{v}+\frac{\partial \bar{y}}{\partial \bar{x}} \tilde{u}
$$

we obtain the corresponding incompressible equations needed to solve the thermal expansion statement and are given by:

$$
\begin{gathered}
\frac{\partial \bar{u}}{\partial \bar{x}}+\frac{\partial \bar{v}}{\partial \bar{y}}=0 \\
\bar{u} \frac{\mathrm{d} \bar{u}}{\partial \bar{x}}+\bar{v} \frac{\partial \bar{u}}{\partial \bar{y}}=-\frac{1}{\rho} \frac{\mathrm{d} \tilde{p}}{\mathrm{~d} \bar{x}}+\frac{\partial}{\partial \bar{y}}\left(\frac{\partial \bar{u}}{\partial \bar{y}}\right) \\
\bar{u} \frac{\partial T}{\partial \bar{x}}+\bar{v} \frac{\partial T}{\partial \bar{y}}=\frac{1}{\operatorname{Pr}} \frac{\partial}{\partial \bar{y}}\left(\frac{\partial T}{\partial \bar{y}}\right)
\end{gathered}
$$




$$
\rho T=1, \quad \rho \mu=1
$$

with the following boundary conditions

$$
\begin{array}{r}
\text { at } \bar{y}=0: \quad \bar{u}=\bar{v}=0, \quad T=1+\left(T_{\mathrm{w}}-1\right) H(\bar{x}) \\
\bar{x} \rightarrow-\infty: \quad \bar{u} \rightarrow \bar{y}, \quad T \rightarrow 1 \\
\bar{y} \rightarrow \infty: \quad \bar{u} \rightarrow \bar{y}+\tilde{A}(\bar{x})+\int_{0}^{\infty}(T-1) \mathrm{d} \bar{y} \\
\bar{x} \rightarrow \infty: \quad \tilde{p} \rightarrow 0, \quad \bar{u} \rightarrow(x-1)^{1 / 3} F_{0}^{\prime}(\eta), \quad T \rightarrow G_{0}
\end{array}
$$

together with

$$
\tilde{p}(\bar{x})=\frac{1}{\pi} \oint_{-\infty}^{\infty} \frac{\tilde{A}\left(\bar{x}_{1}\right) \mathrm{d} \bar{x}_{1}}{\bar{x}-\bar{x}_{1}}
$$

where $F_{0}, y, G_{0}$ satisfy the equations (16)-(18).

\section{LINEARIZED THEORY FOR $T_{\mathrm{w}} \rightarrow 1$}

Defining $\theta_{\mathrm{w}}$ as $\theta_{\mathrm{w}}=T_{\mathrm{w}}-1 \ll 1$, the equations can be linearized around the undisturbed boundary layer profile by expanding the flow variables as follows:

$$
\begin{gathered}
\bar{u}=\bar{y}+0_{\mathrm{w}} \hat{u}_{0}+\cdots, \quad \bar{v}=\theta_{\mathrm{w}} \hat{v}_{0}+\cdots \\
\tilde{p}=0_{\mathrm{w}} \hat{p}_{0}+\cdots, \quad \tilde{A}=\theta_{\mathrm{w}} \hat{A}_{0}+\cdots \\
\rho=1-\theta_{\mathrm{w}} \hat{T}_{0}, \quad \mu=1+\theta_{\mathrm{w}} \hat{T}_{0}+\cdots \\
T=1+\theta_{\mathrm{w}} \hat{T}_{0}+\theta_{\mathrm{w}}^{2} \hat{T}_{1}, \quad \tau=1+\theta_{\mathrm{w}} \hat{\tau}_{0}+\cdots
\end{gathered}
$$

where $\tau$ corresponds to the nondimensional skin friction defined by $\tau=\mathrm{d} \vec{u} / \mathrm{d} \bar{y}$. It is relevant to pay attention that the expansion proposed for the temperature includes terms of order $\theta_{\mathrm{w}}^{2}$. It is required because in this linear theory the thermal expansion process produces significant changes in the temperature profile only within this order. Otherwise, terms of order $\theta_{w}$ in particular for the temperature profile retains only the well-known Lighthill's solution for the Nusselt number. Therefore, the linear nondimensional governing equations are then transformed to:

$$
\begin{gathered}
\frac{\partial \bar{u}_{0}}{\partial \bar{x}}+\frac{\partial \hat{v}_{0}}{\partial \bar{y}}=0 \\
\bar{y} \frac{\partial \hat{u}_{0}}{\partial \bar{x}}+\hat{v}_{0}=-\frac{\mathrm{d} \hat{p}_{0}}{\mathrm{~d} \bar{x}}+\frac{\partial^{2} \hat{u}_{0}}{\partial \bar{y}^{2}} \\
\bar{y} \frac{\partial \hat{T}_{0}}{\partial \bar{x}}=\frac{1}{\operatorname{Pr}} \frac{\partial^{2} \hat{T}_{0}}{\partial \bar{y}^{2}} \\
\bar{y} \frac{\partial \hat{T}_{1}}{\partial \bar{x}}+\hat{u}_{0} \frac{\partial \hat{T}_{0}}{\partial \bar{x}}+\hat{v}_{0} \frac{\partial \hat{T}_{0}}{\partial \bar{y}}=\frac{1}{\operatorname{Pr}} \frac{\partial^{2} \hat{T}_{1}}{\partial \bar{y}^{2}}
\end{gathered}
$$

with the boundary conditions:

$$
\begin{gathered}
\bar{y}=0: \quad \hat{u}_{0}=\hat{v}_{0}=0, \quad \hat{T}_{0}=H(\bar{x}), \quad \hat{T}_{1}=0 \\
\bar{x} \rightarrow-\infty: \quad \hat{u}_{0} \rightarrow 0 \\
\bar{y} \rightarrow \infty: \quad \hat{u}_{0} \rightarrow \hat{A}_{0}+\int_{0}^{\infty} \hat{T}_{0}(\bar{x}, \bar{y}) \mathrm{d} \bar{y} \\
\hat{T}_{0} \rightarrow 0, \quad \hat{T}_{1} \rightarrow 0
\end{gathered}
$$

$$
\begin{gathered}
\bar{x} \rightarrow \infty: \quad \hat{u}_{0} \rightarrow 0 \\
\hat{T}_{0} \rightarrow 0 \\
\hat{T}_{1} \rightarrow 0
\end{gathered}
$$

and

$$
\hat{p}_{0}=\frac{1}{\pi} \oint_{\infty}^{\infty} \frac{A_{0}^{\prime}\left(\bar{x}_{1}\right) \mathrm{d} \bar{x}_{1}}{\bar{x}-\bar{x}_{1}} .
$$

The first step is to obtain the solution up to term of order $\theta_{\mathrm{w}}$. In this case, the energy equation decouples from the continuity and momentum equations. Thereafter, we proceed to include the correction to the Lighthill solution for the Nusselt number within the formalism of Triple-Deck theory. Therefore, from equation (34) we can obtain a self-similar solution for $\hat{T}_{0}$ after some manipulations given by:

$$
\hat{T}_{0}=\frac{\Gamma\left(1 / 3, \frac{P r}{9 \bar{x}} \bar{y}^{-3}\right)}{\Gamma(1 / 3)} H(\bar{x})
$$

where $\Gamma\left(1 / 3, \operatorname{Pr} \bar{y}^{3} / 9 \bar{x}\right)$ represents the incomplete gamma function. The issue of $\hat{T}_{0}$ serve us to complete the momentum boundary layer problem which it is now reduced to:

$$
\begin{gathered}
\partial \hat{u}_{0}+\frac{\partial \hat{v}_{0}}{\partial \bar{y}}=0 \\
\partial \bar{x} \\
\bar{y} \frac{\partial \hat{u}_{0}}{\partial \bar{x}}+\hat{v}_{0}=-\frac{\mathrm{d} \hat{p}_{0}}{\mathrm{~d} \bar{x}}+\frac{\partial^{2} \hat{u}_{0}}{\partial \bar{y}^{2}} .
\end{gathered}
$$

The boundary condition for $\hat{u}_{0}$ as $y \rightarrow \infty$ is now given by

$$
\hat{u}_{0} \rightarrow \hat{A}_{0}(\bar{x})+\frac{9^{1 / 3} \Gamma(2 / 3) \bar{x}^{1 / 3}}{\Gamma(1 / 3) P r^{1 / 3}} H(\bar{x})
$$

leaving the other boundary conditions unaltered. The solution now can be obtained using the Fourier transform with respect to $\bar{x}$. Denoting the variables in the Fourier space by subindex F, wc obtain the following system of equations :

$$
i \omega y \frac{\partial u_{\mathrm{r}}}{\partial \bar{y}}=\frac{\partial^{3} u_{\mathrm{F}}}{\partial \bar{y}^{3}}
$$

with the following boundary conditions :

$$
\begin{gathered}
\bar{y}=0: \quad u_{\mathrm{F}}=v_{\mathrm{F}}=0 \\
P_{\mathrm{F}}=|\omega| A_{\mathrm{F}} \text { (interaction condition) } \\
\bar{y} \rightarrow \infty: \quad u_{\mathrm{F}} \rightarrow A_{\mathrm{F}}+\frac{9^{1 / 3} \Gamma(2 / 3)}{\operatorname{Pr}^{1 / 3} \Gamma(1 / 3)} \frac{\Gamma(4 / 3)}{2 \pi i^{4 / 3} \omega^{4 / 3}}
\end{gathered}
$$

for any value of the wave number $\omega$. Equation (41) represents the well-known Airy equation for the first derivative of $u_{\mathrm{F}}$. Specifically, for this case the solution is given by

$$
\left.\frac{\partial u_{\mathrm{r}}}{\partial \bar{y}}=B(\omega) A_{\mathrm{i}}[(i \omega))^{1 / 3} \bar{y}\right]
$$

where $A_{\mathrm{i}}$ is Airy's function and $B(\omega)$ can be found 
through the use of the boundary conditions (42) and (43), giving

$$
B(\omega)=\left\{\begin{array}{cl}
\frac{3 \pi S(4 / 3) \omega^{1 / 3}}{2 \pi i^{2 / 3}\left(\Lambda^{4 / 3}+i^{1 / 3} \omega^{4 / 3}\right)} & \text { if } \omega>0 \\
-3\left[\leqslant \Gamma(4 / 3) \omega^{1 / 3}\right. & \text { if } \omega<0 \\
2 \pi i^{2 / 3}\left(\Lambda^{4 / 3}-i^{1 / 3} \omega^{4 / 3}\right) &
\end{array}\right.
$$

with $\Lambda=\left[-3 A^{\prime}{ }_{1}(0)\right]^{3 / 4}=0.8272$ and $\mathbb{K}=9^{1 / 3} \Gamma(2 / 3)$ $\operatorname{Pr}^{1 / 3} \Gamma(1 / 3)$. Using the Fourier inversion theorem, we obtain the pressure distribution, skin friction and displacement thickness which are given by:

$$
\begin{aligned}
& \tilde{p}(\bar{x})=\frac{\mathbb{k} 0_{\mathrm{w}} \Gamma(4 / 3) \Lambda^{2 / 3}}{2 \pi}\left\{\int_{0}^{x} \frac{\left[\left(1+\sqrt{\left.3 s^{4 / 3}\right) \cos (\Lambda s \tilde{x})}\right.\right.}{s^{1 / 3}\left(1+\sqrt{3 s^{4 / 3}+s^{8 / 3}}\right)}\right. \\
& \left.-\frac{\left.\left(\sqrt{3}+s^{4 / 3}\right) \sin (\Lambda s \bar{x})\right]}{s^{1 / 3}\left(1+\sqrt{3} s^{4 / 3}+s^{8 / 3}\right)} \mathrm{d} s\right\} \\
& \tau-1=\frac{3 \mathbb{K} \Gamma(4 / 3) 0_{\mathrm{w}}}{2 \pi A_{i}(0)} \\
& \times \int_{0}^{\infty} \frac{\left[s^{1 / 3} \cos (\Lambda s \bar{x})+\left(\sqrt{3}+2 s^{4 / 3}\right) \sin (\Lambda s \bar{x})\right] \mathrm{d} s}{\left(1+\sqrt{3 s^{4 / 3}+s^{8 / 3}}\right)}
\end{aligned}
$$

$$
\left.\begin{array}{l}
\tilde{A}(\bar{x})=-0_{w} \mathbb{R}\left\{\bar{x}^{1 / 3} H(\bar{x})-\frac{\Gamma(4 / 3)}{2 \pi \Lambda^{1 / 3}}\right. \\
\times \int_{0}^{\infty}\left[2\left(1+\frac{\sqrt{3}}{2} s^{4 / 3}\right) \sin (\Lambda s \bar{x})-s^{4 / 3} \cos (\Lambda s \bar{x})\right] \mathrm{d} s \\
1+\sqrt{3} s^{4 / 3}+s^{8 / 3}
\end{array}\right\}
$$

where all the equations are valid for positive and negative values of $\bar{x}$ and the variable $s$ is related to $\omega$ through $s=\omega / \Lambda$. Clearly, the different forms of solutions for $\bar{x}<0$ and $\bar{x}>0$ correspond to closing the contour of integration in $\operatorname{Im} \omega>0$ and $\operatorname{Im} \omega<0$, respectively. The asymptotic behavior for the main variables for $\bar{x} \rightarrow \pm \infty$, obtained from equations (46), (47) and (48), are

$$
\begin{aligned}
& \tilde{A}(\bar{x}) \sim-\mathbb{K} 0_{\mathrm{w}}\left\{\frac{\Gamma(4 / 3)}{\pi \Lambda^{4 / 3}} \frac{1}{( \pm \bar{x})}+( \pm \bar{x})^{1 / 3} H(\bar{x})+\cdots\right\}, \\
& \tilde{x} \rightarrow \pm \infty \\
& \tilde{p}(\bar{x}) \sim \begin{cases}-(3)^{1 / 2} \ll 0,0_{w} / \bar{x}^{2 / 3}+\cdots, & \text { if } \bar{x} \rightarrow \infty \\
2(3)^{1 / 2} \ll 0_{w} /(-x)^{2 / 3}+\cdots, & \text { if } \bar{x} \rightarrow-\infty\end{cases} \\
& \tilde{\tau}-1 \sim\left\{\begin{array}{l}
\theta_{w} \mathbb{K} \Gamma(4 / 3) A_{\mathrm{i}}(0) / \\
3^{1 / 2} \Gamma(2 / 3)(\Lambda \bar{x})^{4 / 3}+\cdots, \quad \text { if } \bar{x} \rightarrow \infty \\
-20_{w} \mathbb{R} \Gamma(4 / 3) A_{\mathrm{i}}(0) / \\
3^{1 / 2} \Gamma(2 / 3)(-\Lambda \bar{x})^{4 / 3}+\cdots, \quad \text { if } \bar{x} \rightarrow-\infty
\end{array}\right.
\end{aligned}
$$

these asymptotic relationships agree with that obtained with the Blasius solution as $\bar{x} \rightarrow-\infty$ and to the modified solution as $\bar{x} \rightarrow \infty$, as shown by equations (22). In both limits the pressure reaches asymptotically the free stream value. The nondimensional skin friction tends also to unity in both limits, which in this case are exactly the same because of the introduction of the Howarth-Dorodnitzyn transformation. On the other hand for $|\bar{x}| \rightarrow 0$, the asymptotic solution for the nondimensional pressure and the displacement thickness are given by

$$
\begin{aligned}
& \tilde{p}(\bar{x}) \sim 3.12336 \frac{\ll \theta_{\mathrm{w}} \Gamma(4 / 3) \Lambda^{2 / 3}}{2 \pi}+O\left(\bar{x}^{2 / 3}\right) \\
& \tilde{A}(\bar{x}) \sim-2.9348 \frac{\llbracket \theta_{\mathrm{w}} \Gamma(4 / 3)}{-2 \pi \Lambda^{1 / 3}}+O(\bar{x})
\end{aligned}
$$

while the skin friction jumps from $1-0.37310_{\mathrm{w}} / \mathrm{Pr}^{1 / 3}$ at $\bar{x}=0^{-}$to $1+0.6218 \theta_{\mathrm{w}} / \mathrm{Pr}^{1 / 3}$ at $\tilde{x}=0^{+}$. The jump of the skin friction at $\bar{x}=0$ and the jump in the pressure gradient at the same position are a result of the existence of the central region not resolved by the Triple-Deck, the structure of which is not considered in the present analysis. However, the singularity in the displacement thickness gradient has been removed. Using this linearized theory we can obtain a critical temperature ratio for separation $(\tau=0)$, as

$$
\left(\theta_{\mathrm{w}}\right)_{\mathrm{c}}=2.68025 \mathrm{Pr}^{1 / 3}
$$

or

$$
\left(T_{w}\right)_{c}=1+2.68025 \operatorname{Pr}^{1 / 3}=3.391 \text { for } \operatorname{Pr}=0.71
$$

which is not very far from that obtained using the nonlinear numerical calculation $\left(T_{\mathrm{w}}\right)_{\mathrm{c}} \approx 3$, to be shown in the next section. This critical temperature ratio as expected increases with the Prandtl number because the cxpansion effects are reduced as the Prandtl number increases. In order to conclude this section, we develop a solution for the thermal problem which is required to modify the Lighthill solution for the Nusselt number [14]. This complementary analysis confirms the importance that the Triple-Deck structure introduces, through the thermal expansion which provides a higher order correction of the heat transfer. Therefore, in order to deduce the role of the thermal expansion within the lower-deck, we need to obtain the temperature correction of $\operatorname{order} \theta_{\mathrm{w}}^{2}, \hat{T}_{1}$, by solving equation (35) and the corresponding boundary conditions. Using the Lighthill approximation, by introducing the following appropriate coordinate, $\sigma=\bar{y} \mathrm{Pr}^{1 / 3}$, equations (32), (33) and (35) transform to

$$
\begin{gathered}
\frac{\partial \hat{u}_{0}}{\partial \bar{x}}+\operatorname{Pr}^{1 / 3} \frac{\partial \hat{v}_{0}}{\partial \sigma}=0 \\
\frac{\partial \hat{\tau}_{0}}{\operatorname{Pr} \hat{\partial} \bar{x}}=\frac{\partial^{2} \hat{\tau}_{0}}{\hat{\partial} \sigma^{2}}
\end{gathered}
$$


$\frac{1}{\operatorname{Pr}^{1 / 3}} \frac{\partial^{2} \hat{T}_{1}}{\partial \sigma^{2}}-\frac{\sigma}{\operatorname{Pr}^{1 / 3}} \frac{\partial \hat{T}_{1}}{\partial \bar{x}}=\hat{u}_{0} \frac{\partial \hat{T}_{0}}{\partial \bar{x}}$

$+\hat{v}_{0} \operatorname{Pr}^{1 / 3} \frac{\partial \hat{T}_{0}}{\partial \sigma}$

where

$$
\hat{T}_{0}=\frac{\Gamma\left(1 / 3, \sigma^{3} / 9 \bar{x}\right)}{\Gamma(1 / 3)} H(\bar{x})
$$

leaving the restant boundary conditions unaltered. The parabolic equations can be solved easily and the results are given by

$$
\begin{gathered}
\hat{u}_{0}=\left\{\frac{\Gamma(1 / 3)}{\Gamma(2 / 3) \operatorname{Pr}^{1 / 3}} \hat{A}(\bar{x})+\frac{H(\bar{x})}{\operatorname{Pr}^{2 / 3}}\right\} \sigma \\
\hat{v}_{0}=-\frac{\Gamma(1 / 3)}{2 \Gamma(2 / 3)}\left\{\frac{(9 \bar{x})^{1 / 3} \hat{A}^{\prime}(\bar{x})-3 \hat{A}(\bar{x})(9 \bar{x})^{-2 / 3}}{(9 \bar{x})^{2 / 3}}\right\} \\
\times \frac{\sigma}{\operatorname{Pr}^{2 / 3}}-\frac{\delta(\bar{x}) \sigma^{2}}{2 \operatorname{Pr}}
\end{gathered}
$$

where $\delta(\bar{x})$ is the Dirac delta function. Clearly, within this approximation the velocity profiles $\hat{u}_{0}$ and $\hat{v}_{0}$ appear linear and quadratic functions of the coordinate $\sigma$, respectively. On the other hand, the displacement thickness $\hat{A}(\bar{x})$ is provided by the solution obtained with the Fourier transform, i.c. equation (48). The specific form of the dependence of $\hat{u}_{0}$ and $\hat{v}_{0}$ on $\sigma$ arises from the fact that the thermal boundary layer grows downstream of the momentum boundary layer. Therefore, the equation for $\hat{T}_{1}$, after substituting $\hat{u}_{0}, \hat{v}_{0}$ and $\hat{T}_{0}$ in terms of $\sigma$, is transformed to :

$$
\begin{aligned}
& \frac{\partial^{2} \hat{T}_{1}}{\partial \sigma^{2}}-\sigma \frac{\partial \hat{T}_{1}}{\partial \bar{x}}=\frac{\Gamma\left(1 / 3, s^{3} / 9 \bar{x}\right) \delta(\bar{x}) \operatorname{Pr}^{1 / 3}}{\Gamma(1 / 3)} \\
& \times\left\{\frac{\Gamma(1 / 3) \hat{A}(\bar{x})}{\Gamma(2 / 3) P r^{1 / 3}(9 \bar{x})^{1 / 3}}+\frac{H(\bar{x})}{P r^{2 / 3}}\right\} \sigma+\frac{P r^{1 / 3} \mathrm{e}^{-\sigma^{3} / 9 \bar{x}}}{\Gamma(1 / 3)(9 \bar{x})^{1 / 3}} \\
& \times H(\bar{x})\left\{\frac{9 \sigma^{2}}{9 \bar{x}}\left[\frac{\Gamma(1 / 3)}{\Gamma(2 / 3) \operatorname{Pr}^{1 / 3}} \frac{\hat{A}(\bar{x})}{(9 \bar{x})^{1 / 3}}+\frac{H(\bar{x})}{P r^{2 / 3}}\right]\right. \\
& -3 \operatorname{Pr}^{1 / 3}\left[\frac{-\Gamma(1 / 3)}{\Gamma(2 / 3) \operatorname{Pr}^{2 / 3}}\right. \\
& \times\left(\frac{(9 \bar{x})^{1 / 3}}{\hat{A}^{\prime}(\bar{x})-3 \hat{A}(\bar{x})(9 \bar{x})^{-2 / 3}} \frac{\sigma^{2}}{(9 \bar{x})^{2 / 3}}\right)_{2} \\
& \left.\left.-\frac{\delta(\bar{x})}{\operatorname{Pr}} \frac{\sigma^{2}}{2}\right]\right\}
\end{aligned}
$$

with the boundary conditions :

$$
\begin{array}{llll}
\sigma=0: & \hat{T}_{1}=0, & \sigma \rightarrow \infty & \hat{T}_{1}=0 \\
\bar{x}<0: & \hat{T}_{1}=0, & \bar{x} \rightarrow \infty & \hat{T}_{1}=0 .
\end{array}
$$

In order to simplify the above equation, it should be observed that $\delta(\bar{x})$ terms are irrelevant for all range of $\bar{x}$, because it can be shown that for finite values of $\bar{x}$ and also for values of $\bar{x}$ tending to zero, those terms are identically zero, due to the properties of the Dirac delta function and similarly to the asymptotic expansions for the gamma incomplete function. Therefore, the restant equation of $\hat{T}_{1}$ can be solved if we assume a solution $\hat{T}_{1}=\sigma \mathrm{e}^{-\sigma^{3} / 9 \bar{x}} \phi(\bar{x}) H(\bar{x})$. The resulting ordinary differential equation for $\phi(\bar{x})$ is then given as

$$
\begin{aligned}
\frac{\mathrm{d} \phi}{\mathrm{d} \bar{x}} & +\frac{12 \phi}{9 \bar{x}}=-\frac{9}{2 \Gamma(2 / 3)(9 \bar{x})^{5 / 3}} \\
& \times\left\{\hat{A}(\bar{x})+\frac{\bar{x} \hat{A}^{\prime}(\bar{x})}{3}+\frac{2 \Gamma(2 / 3)}{\Gamma(1 / 3)} \frac{(9 \bar{x})^{1 / 3} H(\bar{x})}{P r^{1 / 3}}\right\}
\end{aligned}
$$

with the condition :

$$
\phi=0 \text { for } \bar{x} \rightarrow \infty .
$$

The solution for $\hat{T}_{1}$ is then given as

$$
\begin{aligned}
& \hat{T}_{1}=-\frac{9}{2} \frac{\sigma}{\Gamma(2 / 3)} \mathrm{e}^{-\left(\sigma^{3} / 9 \bar{x}\right)} \int^{\bar{x})^{4 / 3}} \int_{0}^{\bar{x}} \\
& \quad \times \frac{\left\{\hat{A}(\bar{x})+\frac{\bar{x} \hat{A}^{\prime}(\bar{x})}{3}+\frac{2 \Gamma(2 / 3)}{\Gamma(1 / 3)}\left(\frac{9 \bar{x}}{P r}\right)^{1 / 3} H(\bar{x})\right\}}{(9 \bar{x})^{1 / 3}} \mathrm{~d} \bar{x} H(\bar{x}) .
\end{aligned}
$$

Finally, the comparison between the modified Nusselt number and the Lighthill solution, after some manipulations, can be written as

$$
\begin{aligned}
& N u^{*}=N u / N u_{\mathrm{L}}-1=\frac{\Gamma(1 / 3) \theta_{\mathrm{w}}}{2 \Gamma(2 / 3)} \\
& \times\left\{\frac{\hat{A}(\bar{x})}{(9 \bar{x})^{1 / 3}}+\frac{2}{3} \frac{\Gamma(2 / 3)}{3 \Gamma(1 / 3) \operatorname{Pr}}+\frac{7}{27 \bar{x}} \int_{0}^{\bar{x}} \frac{\hat{A}(\bar{x}) \mathrm{d} \bar{x}}{(9 \bar{x})^{1 / 3}}\right\} H(\bar{x})
\end{aligned}
$$

where $N u_{\mathrm{L}}$ corresponds to the asymptotic solution due to Lighthill [14] given by

$$
N u_{\mathrm{L}}=\left(\frac{3^{1 / 3} \rho_{\mathrm{W}} \operatorname{Pr}^{1 / 3} R^{1 / 2} x}{\Gamma(1 / 3)} \frac{(x-1)^{1 / 3}}{(x-1)}\right)
$$

with $\hat{A}(\bar{x})$ given by equation (48). The asymptotic solution for the Nusselt number is given by

$$
N u \sim\left(1+\frac{\Gamma(1 / 3)}{2 \Gamma(2 / 3)} \theta_{w}\right) \frac{1}{\bar{x}^{1 / 3}}
$$

for $\bar{x} \rightarrow 0$ and the corresponding $N u \sim \bar{x}^{1 / 3}$ when $\bar{x} \rightarrow \infty$.

\section{NONLINEAR ANALYSIS}

It is clear that for values of $T_{\mathrm{w}}$ not close to one, the complete solution of the problem defined by equations (25)-(30) requires a relatively sophisticated numerical analysis. Diverse numerical techniques have been implemented and discussed in the past in order to solve interaction problems within the framework of the Triple-Deck structure. However, for subsonic flow, the feedback mechanism due to the 
outer flow introduces the interaction law (31), which is global in character. In this paper we use the numerical scheme developed by Veldman and Dijkstra [7], adapted for the present thermal expansion process.

The method used in order to solve equations (25) (30) can be resumed under the following general steps : By using the linear solution given by equations (46) and (48) for the pressure and displacement thickness distributions, it is possible to produce initial values of the profiles which can be used to solve simultaneously the Hilbert integral and the boundary layer equations. The previous known values at any station are incorporated after some iterations for each station, input to the next station. The problem should be completed with a global convergence criterion. Here, the parameter chosen to implement this criterion is fundamentally the displacement thickness $\tilde{A}(\bar{X})$ and it is imposed to be

$$
\left|A_{\text {ncw }}-A_{\text {old }}\right|<10^{-6}
$$

which gives about five figure accuracy for most predicted quantities. Therefore, comparing the new $A(\bar{x})$ with the $A(\bar{x})$ from the previous iteration determines if the program has converged. If not, $A(\bar{x})$ is replaced according to the formula

$$
A(\vec{x})=\mathbb{B} A_{\text {old }}+(1-\mathbb{B}) A_{\text {new }}
$$

until the differences between succeeding $A(\bar{x})$ iterates is less than $10^{-6}$. It has been found by trial and error that $\mathbb{B}=0.85$ will produce a convergent iteration schemc. For $\mathbb{B}=0.5$, this global criterion does not converge and strong oscillations mainly at the skin friction and the heat flux are presented. The parabolic equations (25)-(27), together with the corresponding boundary conditions are numerically solved using the implicit method due to Keller [18], which is widely known in the literature as the Box Method. This method has several very desirable features which make it appropriate for the solution of all kind of parabolic partial differential equations. The main features of this method can be resumed as follows: second order accuracy with arbitrary nonuniform $\bar{x}$ and $\bar{y}$ spacing; allows very rapid $\bar{x}$ variations and also permits easy programming of the solution of large numbers of coupled equations. The solution by this method can be obtained by the following four steps :

(1) Reduce the equations at the lower-deck to a first-order system.

(2) Write difference equations using central differences.

(3) Linearize the resulting algebraic equations through Newton's method and write them in a matrixvector form.

(4) Solve the linear system by the block-tridiagonaleliminations method.

If we follow the above steps, previously having intro duced the change of variables $\bar{u}=\partial \psi / \partial \bar{y}, \bar{v}=-\partial \psi / \partial \bar{x}$ into the governing equations (25)-(27), it is possible to define new dependent variables $\mathbb{U}(\bar{x}, \bar{y}), \mathbb{V}(\bar{x}, \bar{y})$ and $\pi$, so that the transformed momentum and energy equations can be written as

$$
\begin{gathered}
\frac{\partial \psi}{\partial \bar{y}}=\mathbb{U} \\
\frac{\partial \bar{u}}{\partial \bar{y}}=\mathbb{V} \\
\mu \frac{\partial \mathbb{V}}{\partial \bar{y}}=\frac{1}{\rho} \frac{\partial \tilde{p}}{\partial \bar{x}}+\mathbb{U} \frac{\partial \mathbb{J}}{\partial \bar{x}}-\frac{\partial \psi}{\partial \bar{x}} \mathbb{V} \\
\frac{\partial T}{\partial \bar{y}}=\mathbb{U} \\
3 \frac{\mu \rho}{\operatorname{Pr}} \frac{\partial \pi}{\partial \bar{y}}=U \frac{\partial T}{\partial \bar{x}}-\frac{\partial \psi}{\partial \bar{x}} \tau .
\end{gathered}
$$

The corresponding boundary conditions except the interaction condition, are to be omitted for simplicity.

\section{RESULTS AND DISCUSSION}

Figures 2-5 show the linear solution for the TripleDeck equations, for the reduced nondimensional pressure $\left(\tilde{p} / \theta_{\mathrm{w}}(\operatorname{Pr} / 0.71)^{1 / 3}\right)$, skin friction $((\tilde{\tau}-1) /$ $\left.\theta_{\mathrm{w}}(\operatorname{Pr} / 0.71)^{1 / 3}\right)$, displacement thickness $\left(\tilde{A} / 0_{\mathrm{w}}(\operatorname{Pr} /\right.$ $0.71)^{1 / 3}$ ) and the reduced Nusselt number $\left(N u^{*}\right)$, respectively. In Fig. 2 we can see how the pressure first increases due to the expansion effects in the lower-deck. This positive pressure gradient has a big influence in regions close to the wall, where the convective terms are rather small. The central region causes a jump in the pressure gradient but not in the pressure itself. For positive values of $\bar{x}$, the pressure decreases strongly reaching values lower than the ambient pressure, increasing asymptotically to this value for large positive values of $\bar{x}$. Figure 3 shows the reduced nondimensional skin friction or shear stress at the wall. For negative values of $\vec{x}$, the disfavorable pressure gradient produces a decreasing shear stress at the wall. From the linear analysis, the critical conditions for separation can be obtained $\tilde{\tau}=0$, giving $\theta_{w_{c}}=2.391$ for $\operatorname{Pr}=0.71$. For positive values of $\bar{x}$, the shear stress jumps to positive values decreasing asymptotically to that given by Blasius as $\bar{x} \rightarrow \infty$. Figure 4 shows the displacement thickness evolution obtained by the linearized theory. Figure 5 shows the modified Nusselt number $N u^{*}$ resulting from comparison with the asymptotic solution by Lighthill. For $N u^{*}=0$ we recover Lighthill's solution. We show that this correction is also singular due to the free interaction with the outer inviscid flow. Strong corrections are obtained for small values of $\bar{x}$.

In relation to the numerical results of the nonlinear governing Triple-Deck equations, all computations for the present algorithm have been performed on an IBM/4381 computer. The base calculations presented here were chosen for a nonuniform grid which has the numerical end point $\bar{x}= \pm 5$ and the outer edge of the lower-deck located at $\bar{y}=8$. Because the grid is 


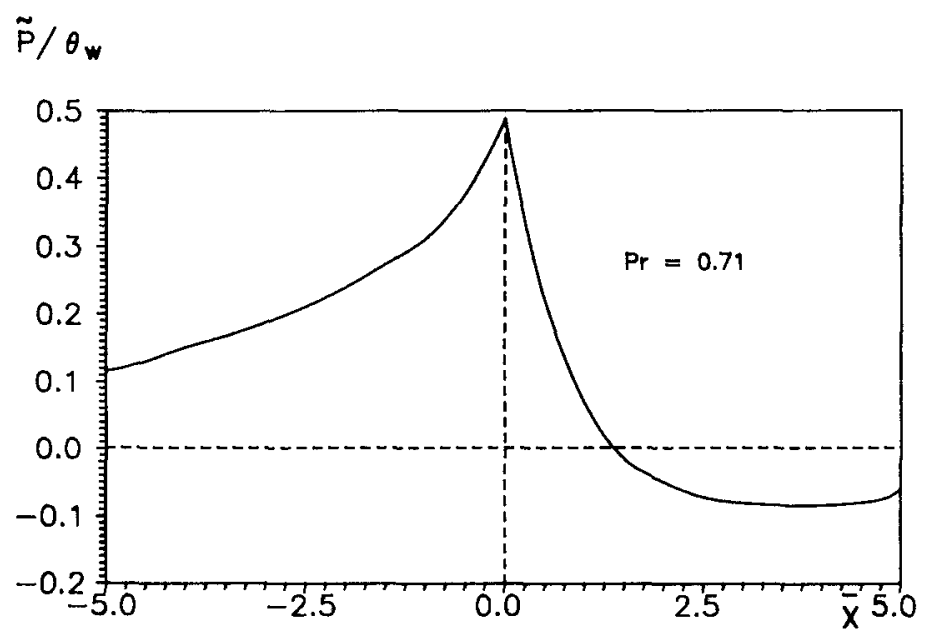

FIG. 2. Results for the pressure distribution dictated by the linearized theory.

$$
(\tilde{\tau}-1) / \theta_{w}
$$

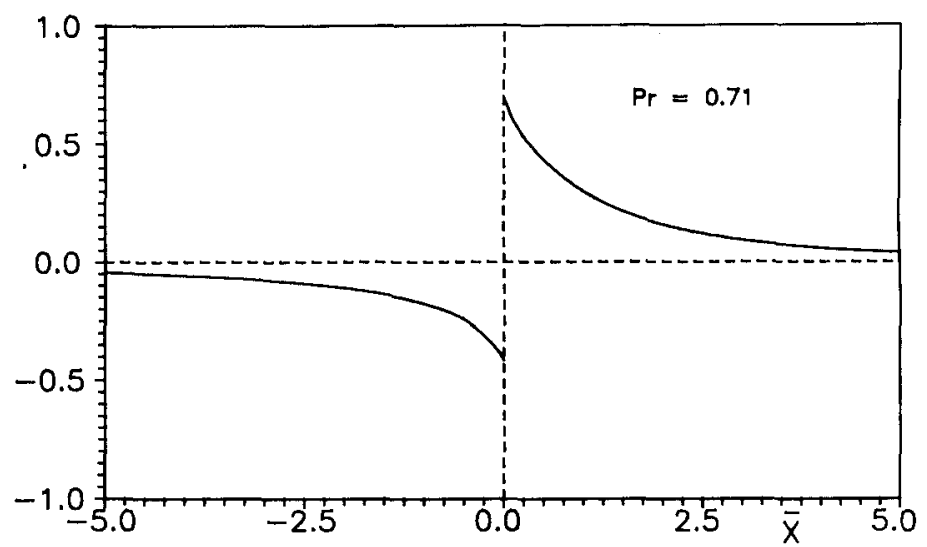

FIG. 3. Skin friction on the wall given by the linearized theory.

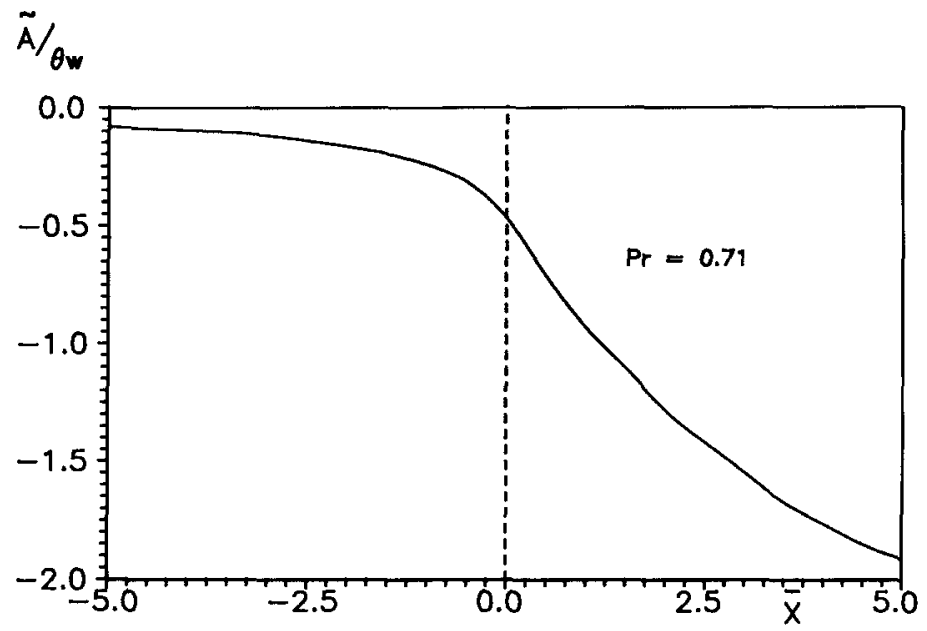

FIG. 4. Displacement thickness given by the linearized theory. 


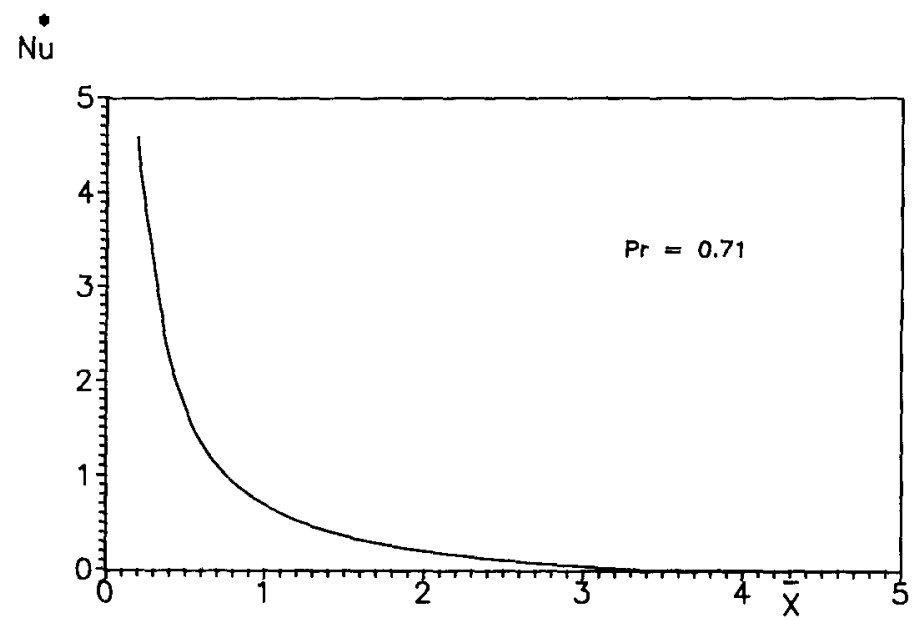

FIG. 5. Modified Nusselt number employing linearized theory.

nonuniform, the initial step sizes for both $\bar{x}$ and $\bar{y}$ were $\Delta \bar{r}=\Delta \bar{y}=0.05$, and also the ratio of lengths of any two adjacent intervals was taken as 1.04 and 1.02 , respectively. We have taken 57 grid points in the transversal coordinate of the lower-deck, which is sufficient for most laminar-flow calculations and for the longitudinal streamwise coordinate we used 80 stations. For a given set of calculations presented here we used a Prandtl number of $P r=0.71$ and the ChapmanRubesin parameter $C=\mu \rho=1$. Figure 6 shows the pressure distribution for several values of the temperature parameter $T_{\mathrm{w}}$. Here, as the value of the wall temperature $T_{\mathrm{w}}$ increases, the expansion process modifies substantially the pressure distribution up to a value of $T_{w}$ close to 3.0, where the increase in pressure distribution which is disfavorable, causes a decrease in skin friction too strong to yield values of skin friction identical to zero, indicating a separation of the lower-deck. This evolution of the skin friction for the corresponding values of $T_{w}$ given above is shown in Fig. 7. For values of $T_{w}$ greater than 3.0 the skin friction goes to zero and the present analysis is no longer valid in order to study this new structure where recirculation and reattachment zones appear. In a similar way as was observed by the pressure distribution, the skin friction reaches asymptotically the Blasius and modified Blasius solution for values of $\bar{x}$ far away from the origin. The displacement thickness is shown in Fig. 8. Clearly, the displacement thickness is practically zero for $\bar{x}<0$ as predicted by the Blasius solution. Otherwise, for values of $\bar{x}>0$ the function $A(\vec{x})$, interpreted as the velocity slip in the higher order at the base of the main-deck, has a significant contribution necessary to cause the pressure disturbances. From the physical point of view, the derivative of $A(\bar{x})$ is the negative of the normal velocity to the plate at the lower edge of the upper-deck and as is shown in Fig. 8, the vertical velocity is not discontinuous at the point where the step temperature takes place. Therefore, the maximum normal velocity occurs immediately after the step change in the wall temperature.

On the other hand, Fig. 9 shows the nondimensional heat flux, $q=\partial T / \partial \bar{y}$, evaluated at the wall, predicted by the strong thermal process for different values of $T_{\mathrm{w}}$. It can be seen from this figure

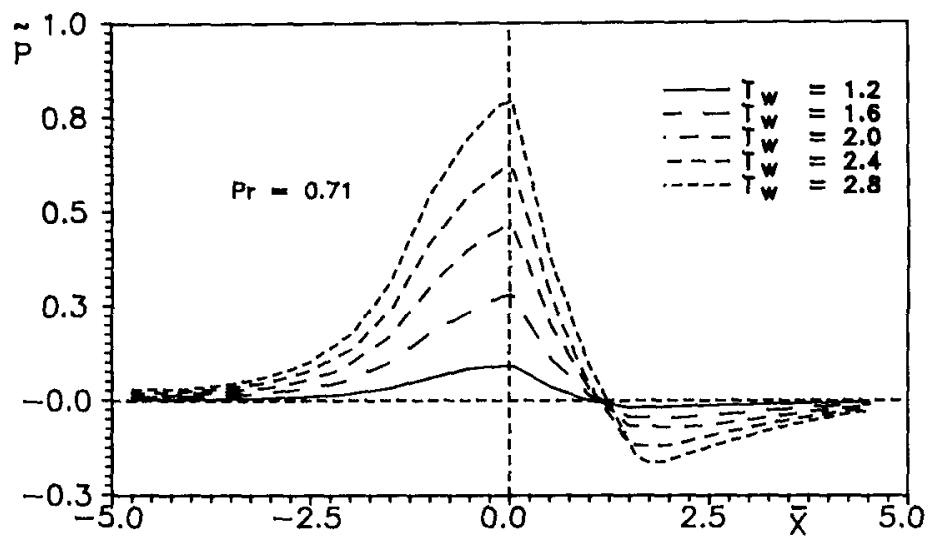

FIG. 6. Pressure distribution for different values of the wall temperature parameter, $T_{\mathrm{w}}$ (numerical calculations). 


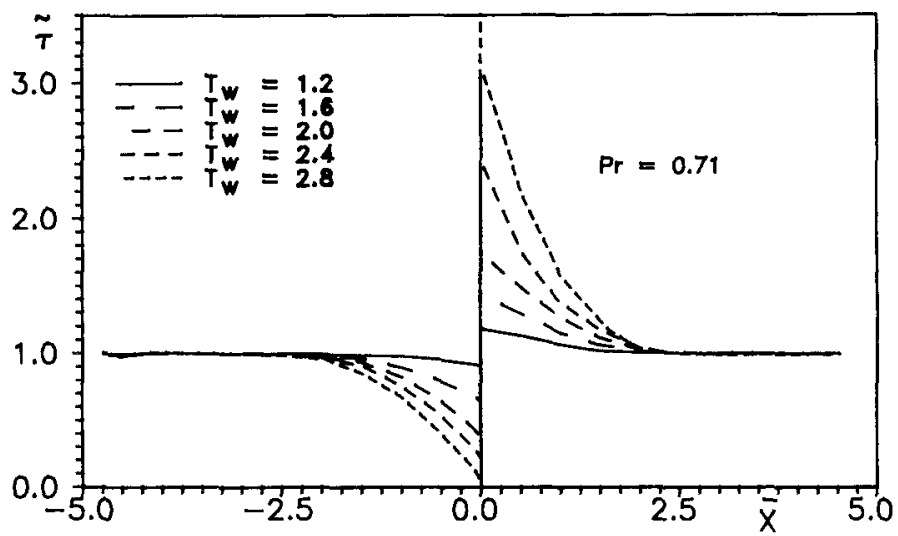

FIG. 7. Skin friction for different values of the wall temperature parameter, $T_{\mathrm{w}}$ (numerical calculations).

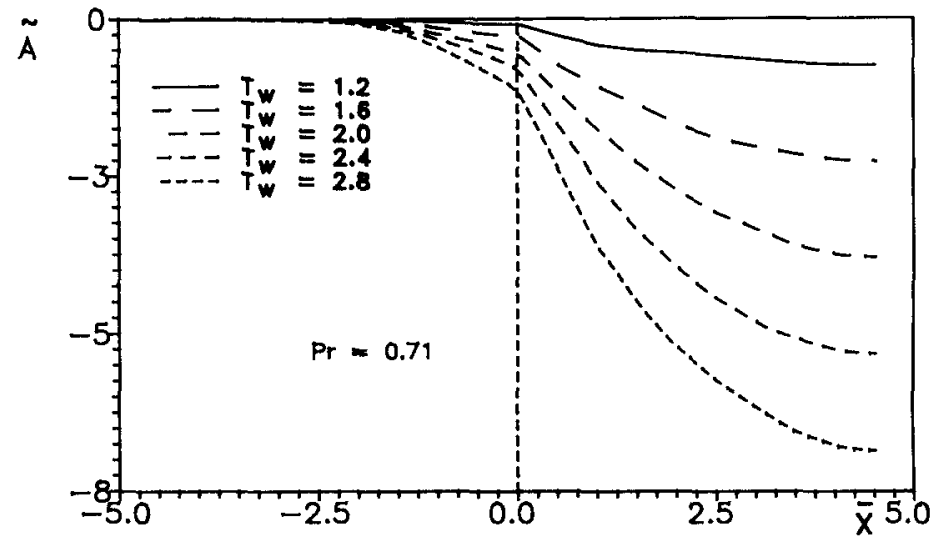

FIG. 8. Displacement thickness for different values of the wall temperature parameter, $T_{\mathrm{w}}$ (numerical calculations).

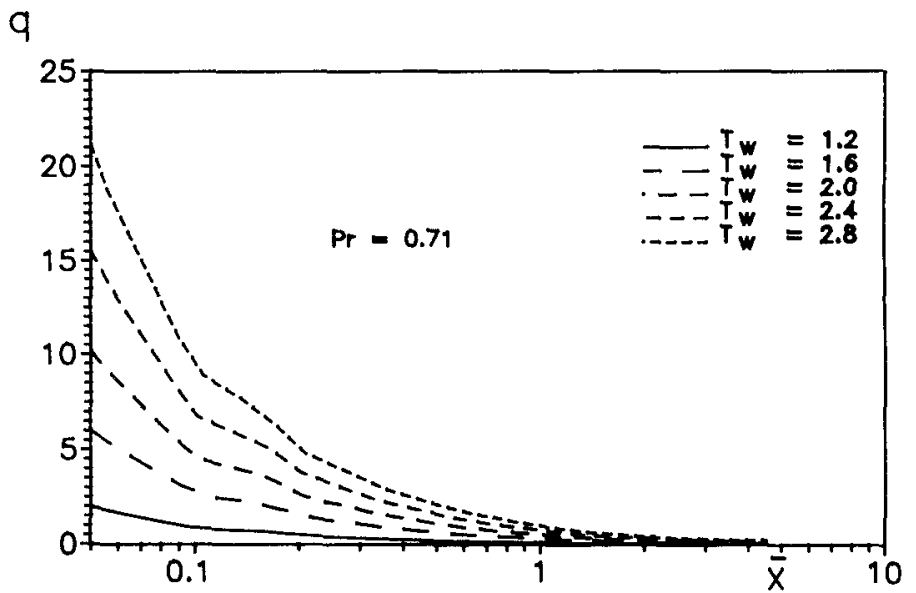

FIG. 9. Heat flux given by $q=\partial T /\left.\partial \bar{y}\right|_{\bar{y}=0}=-\left(T_{\mathrm{w}}-1\right) \lambda^{-3 / 4} R^{-5 / 8} N u / \theta_{\mathrm{w}}\left(1+\lambda^{-5 / 4} R^{\cdots / 8} \bar{x}\right)$ for different values of the wall temperature parameter, $T_{w}$ (numerical calculations).

that when the value of $T_{\mathrm{w}}$ increases, the heat flux increases notably and more specifically close to the origin. Clearly, just at $\bar{x}=0$ the heat flux is singular due to the singular boundary condition. Figures 10 and 11 show the effect of the nonlinear terms on the pressure and the skin friction, respectively. The linear analysis overestimates both the maximum pressure as well as the minimum skin friction. The critical value of $\theta_{\mathrm{w}}$ obtained from the linear analysis is 2.391, for a Prandtl number of 0.71 , while the numerical prediction for separation from the full nonlinear equations was very close to 2 . 


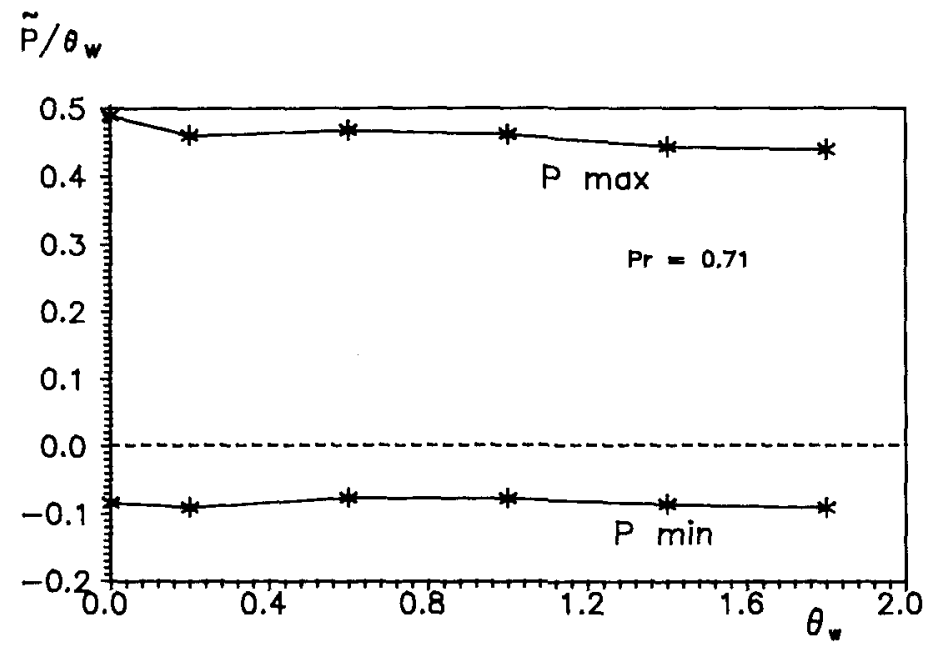

Fig. 10. Comparison between the linear pressure distribution and that predicted for the numerical analysis.

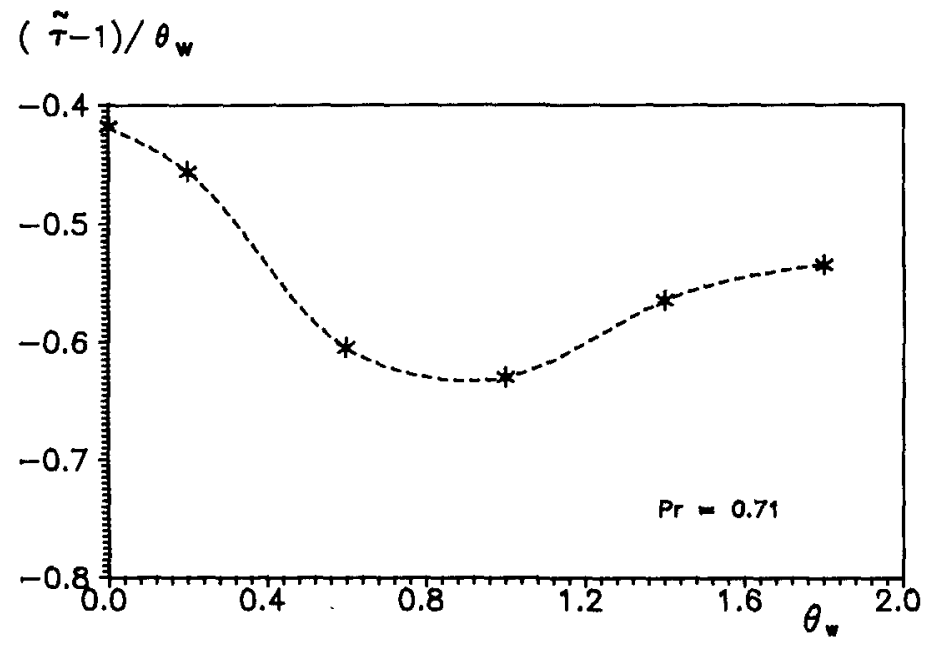

FrG. 11. Comparison between the linear skin friction and that predicted for the numerical analysis.

\section{CONCLUDING REMARKS}

The thermal expansion process for a step change in wall temperature was analysed with the formalism of the Triple-Deck theory. The pressure gradient is disfavorable up to the position of the temperature change, steeply favorable immediately after this position. In t'ie last part, the pressure finally increases asymptotically to the uniform pressure solution. The skin friction, however, decreases from the Blasius value arriving at a zero value if $T_{\mathrm{w}}$ is close to 3.0 , for a Prandtl number $P r=0.71$, causing a separation, where the present analysis is no longer valid. This is because the appearance of a recirculation zone, for values of $T_{\mathrm{w}}$ greater than this critical value. The present analytical model should be modified to include recirculation and reattachment zones. As the separation takes place, heated gas is convected upstream, therefore a cooling process must be established in order to maintain the temperature of the plate at the same level, upstream to the point of temperature change. If, however, an adiabatic boundary condition is applied to this upstream portion of the plate, then after separation takes place, the upstream portion of the plate is heated, reducing the strength of the singularity, until separation disappears. This phenomena could lead eventually to an oscillatory behavior in the boundary layer flow. More work is needed to elucidate these type of interaction problems.

The linear theory predicts the critical value of the temperature ratio $T_{w}$ within an error of $15 \%$ and then can be used in any order of magnitude analysis.

Acknowledgements-The authors thank the Ministerio de Educación y Cultura of Spain for supporting continuous travel funds and for the support of a sabbatical fellowship to C.T.

\section{REFERENCES}

1. K. Stewartson and P. G. Williams, Self-induced separation, Proc. R. Soc. A312, 181-206 (1969).

2. A. F. Messiter, Boundary layer flow near the trailing edge of a flat plate, SIAM J. Appl. Math. 18, 241-257 (1970). 
3. K. Stewartson, D'Alembert's paradox, SIAM Rev. 23, 308-343 (1981).

4. F. T. Smith, On the high Reynolds number theory of laminar flows, IMA J. Appl. Math. 28, 207-281 (1982).

5. K. Stewartson, On laminar boundary layers near corners, Q. J. Appl. Math. 23, 137-152 (1970).

6. F. T. Smith and J. H. Merkin, Triple-deck solutions for subsonic flow past humps, steps, concave or convex corners, and wedged trailing edges, Comput. Fluids 10, 7-25 (1982)

7. A. E. P. Veldman and D. Dijkstra, A fast method to solve incompressible boundary-layer interaction problems, Proc. 7th Int. Conf. Numer. Meth. Fluid Dyn., Stanford, U.S.A. (1980)

8. M. Napolitano and R. E. Messick, On strong slot-injection into a subsonic laminar boundary layer, Comput. Fluids 8, 199-212 (1980).

9. O. F. Burggraf, Asymptotic theory of separation and reattachment of a laminar boundary layer on a compression ramp, AGARD paper 168 on flow separation, Gottingen (1975).

10. A. F. Messiter and A. Liñán, The vertical flat plate in laminar free convection: effects of leading and trailing edges and discontinuous temperature, $Z$. Angew. Math. Phvs. 27, 633-651 (1976).
11. R. Kh. Zeytounian, Les Modèles Asymptotiques de la Mécanique des Fluides II, Lecture Notes in Physics 276. Springer, Berlin (1987)

12. E. M. Sparrow and H. S. Yu, Local non-similarity thermal boundary-layer solutions, J. Heat Transfer 93, 328-334 (1971)

13. T. Cebcei and P. Bradshaw, Physical and Computational Aspects of Convective Heat Transfer. Springer. Berlin (1984).

14. M. J. Lighthill, Contributions to the theory of heat transfer through a laminar boundary layer, Proc. $R$. Soc. A202, 359 (1950)

15. S. Goldstein, Concerning some solutions of the boundary-layer equations in hydrodynamics, Proc. Camb. Phil. Soc. 26, 1-30 (1930)

16. N. Rott and R. J. Hakkinen, Numerical solutions for merging shear flows, Douglas Aircraft Co., Inc., Rept. SM-47 (1965).

17. L. Howarth, Concerning the effect of compressibility on laminar boundary layers and their separation, Proc'. $R$. Soc. A194, 16 (1948).

18. H. B. Keller, A new difference scheme for parabolic equations. In Numerical Solution of Partial Differential Equations (Edited by J. Bramble), Vol. II. Academic Press, New York (1970). 